\title{
Comunicación
}

\section{Efecto del uso de acetato de deslorelina en la inducción de ovulación de yeguas Caballo Peruano de Paso}

\author{
EFFECT OF THE USE OF DESLORELIN ACETATE IN THE INDUCTION OF OVULATION \\ of Peruvian Paso mares
}

Elsa Chávez C. ${ }^{1,3}$, Juan Baltodano T. ${ }^{1}$, Carlos Caballero L. ${ }^{2}$

\section{Resumen}

\begin{abstract}
Se realizó un estudio en 16 yeguas cíclicas Caballo Peruano de Paso de la provincia de Ascope, La Libertad, Perú, entre enero y marzo de 2017, con el fin de evaluar el efecto de la administración de acetato de deslorelina en el tiempo de ovulación y tasa de preñez. Se trabajó con un grupo control $(n=8)$ que no recibió tratamiento y un grupo experimental $(\mathrm{n}=8)$ tratado con $1.75 \mathrm{mg}$ de acetato de deslorelina al observar un folículo dominante de 38-40 mm y un grado 2 a 3 de edema endometrial determinado mediante ultrasonido. Las yeguas fueron inseminadas a las 16-24 h posteriores. El monitoreo mediante ultrasonido se continuó hasta registrar la ovulación y el diagnóstico de preñez se hizo a los 13-15 días pos-ovulación. El tiempo de ovulación fue de $43.13 \pm 4.48$ y $69.00 \pm 8.41 \mathrm{~h}$ para el grupo experimental y control, respectivamente. El 87.5\% (7/8) de las yeguas en el grupo experimental ovularon dentro de las $48 \mathrm{~h}$, mientras que solo el 37.5\% (3/8) del grupo control lo hicieron. La tasa de preñez fue de $87 \%$ (7/8) en el grupo tratado y $50 \%(4 / 8)$ en el grupo sin tratar; en todos los casos sin encontrase diferencias estadísticas significativas.
\end{abstract}

Palabras clave: yeguas; ovulación; acetato de deslorelina

\section{Abstract}

The study was conducted in 16 cyclic mares of the Peruvian Paso breed from the province of Ascope, La Libertad, Peru, between January and March 2017, to evaluate the effect of deslorelin acetate administration on ovulation time and pregnancy rate. There was a control group $(n=8)$ without treatment and an experimental group $(n=8)$ treated

\footnotetext{
${ }^{1}$ Facultad de Ciencias Agrarias, Escuela Profesional de Medicina Veterinaria y Zootecnia, Universidad Privada Antenor Orrego, La Libertad, Perú

${ }^{2}$ Facultad de Ciencias Veterinarias, Universidad Nacional de Cajamarca, Cajamarca, Perú

${ }^{3}$ E-mail: echavezc2@upao.edu.pe
} 
with $1.75 \mathrm{mg}$ of deslorelin acetate when observing a dominant follicle of $38-40 \mathrm{~mm}$ and a grade 2 to 3 of edema endometrial determined by ultrasound. The mares were inseminated at 16-24 $\mathrm{h}$ afterwards. The ultrasound monitoring was continued until ovulation and pregnancy diagnosis was done 13-15 days after ovulation. The ovulation time was 43.13 \pm 4.48 and $69.00 \pm 8.41 \mathrm{~h}$ for the experimental and control group, respectively. Ovulation within 48 h occurred in $87.5 \%$ (7/8) of the mares in the treated group, while only in $37.5 \%$ $(3 / 8)$ of the control group did. The pregnancy rate was $87 \%(7 / 8)$ in the treated group and $50 \%(4 / 8)$ in the control group; in all cases without finding significant statistical differences.

Key words: mares; ovulation; deslorelin acetate

\section{INTRODUCCIÓN}

El caballo es un animal que ha acompañado al hombre a lo largo de su historia y cada vez ha merecido más y mejores atenciones debido a los múltiples beneficios que se han obtenido de él, ya sea para el ejército, la equitación o para los hipódromos, y más aún en aquellos países en los cuales los equinos siguen siendo un apoyo extraordinario para el transporte, como animales de tracción, de carga o para el trabajo del campo en general. No obstante, uno de los problemas que se han enfrentado los criadores de caballos es precisamente la eficiencia en su cría, debido a las inconsistencias reproductivas que presentan, aunado a manejos diversos en sus cuidados (Davies, 2005). Por otra parte, los cambios actuales en la economía mundial han influido de alguna manera en la crianza del caballo, desafiando a los criadores y propietarios, que se han visto obligados a criar menos potrillos o potrancas, a cambio de animales de mayor calidad y valor (Zamudio, 2005; Fragoso, 2007).

Existen muchas diferencias en el ciclo reproductivo entre la yegua y las demás especies. La duración de los ciclos de la yegua es muy variable, al igual que la duración del estro y los niveles hormonales. En ocasiones, ovula en forma independiente respecto a los demás factores ( $\sin$ formación de edema endometrial, cérvix cerrada, etc.), otras ve- ces puede mostrar signos de celo sin llegar a ovular, así como puede ocurrir un celo silente con ovulación. Dichas peculiaridades la convierten en uno de los animales domésticos de más difícil manejo reproductivo (Davies, 2005; Galina y Valencia, 2008).

Por ese motivo, una de las situaciones que más preocupa a los criadores y propietarios de caballos peruanos de paso es poder obtener el máximo rendimiento reproductivo de sus yeguas. En la actualidad se dispone de técnicas de reproducción asistida y se tiene en marcha diversas investigaciones con el objetivo de mejorar su eficiencia reproductiva, sin verse obligados a retirarlas o apartarlas por temporadas (Rodríguez, 2008).

La mayor parte de las investigaciones sobre el control reproductivo en las yeguas se ha centrado en el uso de prostaglandinas (Zamudio, 2005; Fragoso, 2007) y hCG (Yoon, 2012), así como en el GnRH y sus análogos (Barrier-Battut et al., 2001; Camillo et al., 2004). Por otro lado, existen nuevos tratamientos hormonales disponibles (McKinon, 2009), como es el caso del acetato de deslorelina, un análogo superagonista de la $\mathrm{GnRH}$, para el acortamiento del tiempo de ovulación y mejora del índice de preñez, como ha sido reportado en Chile (Maturana, s.f.), EEUU (Blanchar et al., 2002; McCue et al., 2002; Sauberli, 2013), Australia (Finan et al., 2016) y Brasil (Melo et al., 2012), entre otros países. 
Conociendo la eficacia de este análago de la GnRH y debido a la ausencia de trabajos de investigación previos o de antecedentes documentados de su uso en el Perú, es que se evaluó el efecto de la aplicación del acetato de deslorelina en el tiempo de ovulación y porcentaje de preñez en yeguas cíclicas de Caballo Peruano de Paso.

\section{Materiales y Métodos}

El estudio se desarrolló en el Criadero de Caballos Peruanos de Paso de la Ganadería Monte Cristo S.A.C. (Paiján), ubicada en la Provincia de Ascope, La Libertad, Perú, entre enero y marzo de 2017.

Se seleccionaron 16 yeguas, según la disponibilidad de animales que permitió el criadero, entre 4 y 12 años y de similar condición corporal (entre 4 y 6 según la escala de Hennecke et al., 1983) que no presentaron antecedentes de problemas reproductivos como ciclos estrales irregulares, quistes ováricos o endometriales, reabsorción embrionaria, abortos, etc., independientemente de su edad. Todos los animales seleccionados habían tenido como mínimo un parto llegado a término con éxito y no tenían cría al pie.

Las yeguas fueron distribuidas en un grupo control (CO) y en un grupo experimental (EX), cada uno con ocho yeguas. Ambos grupos estuvieron bajo un sistema intensivo de crianza, suministro de agua ad libitum y alimentación a base de chala verde picada y entera, panca seca y pacas de heno de alfalfa. A cada yegua se le asignó un código alfanumérico, según al grupo al que pertenecían.

Las yeguas fueron examinadas 3-4 veces por semana (entre las 09:00 y 17:00 h) mediante ultrasonografía. Se utilizó el ecógrafo SIUI CTS-800 con un transductor lineal de $7 \mathrm{MHz}$ para monitorear el desarrollo folicular hasta la detección de un folículo dominante (FD) de 38 a $40 \mathrm{~mm}$ y un grado de edema endometrial de 2 a 3 (dentro de una escala de 1 a 5, según Samper et al. [2007]). El día del hallazgo de estas estructuras (día 1) se trató a las yeguas EX con $1 \mathrm{ml}$ de deslorelin (Specialized Performance Compounds - LLC, EEUU), vía IM (equivalente a $1.75 \mathrm{mg} /$ animal de acetato de deslorelina), registrándose la fecha y hora de aplicación. Así mismo, en el Día 1 se realizó el primer servicio por inseminación artificial (IA) con semen fresco, tanto a las yeguas del CO como a las yeguas del EX.

Se eligió únicamente a un padrillo para los servicios. La selección se hizo de acuerdo a su registro genealógico, performance en los concursos de los dos últimos años (2015 y 2016) y evaluación de progenie. El semen fue colectado minutos antes de realizar el servicio, mediante el uso de una vagina artificial equina, modelo Missouri, con temperatura en la cámara interna entre 45 y $52{ }^{\circ} \mathrm{C}$. El factor de dilución varió según el volumen de semen filtrado ( $\sin$ gel) obtenido, la concentración de células espermáticas fue determinada mediante hemocitometría con cámara de Neubauer y el porcentaje de motilidad progresiva se observó al microscopio de luz en lámina portaobjeto precalentada $\left(30^{\circ} \mathrm{C}\right)$.

El semen, en promedio, tuvo las siguientes características: volumen $=45 \mathrm{ml}$, concentración de espermatozoides $=350$ millones $/ \mathrm{ml}$, motilidad progresiva $=70 \%$. El factor de dilución fue de 7 ( 1 parte de semen y 6 partes de diluyente). El dilutor empleado fue el E-Z MIXIN CTS (ARS). Se obtuvo un volumen mínimo de dosis inseminante diluida de $30 \mathrm{ml}$ por yegua, lo cual aseguraba una dosis inseminante de $1000 \mathrm{millones} / \mathrm{ml}$ de espermatozoides con motilidad progresiva de $70 \%$ para semen fresco. En la mayoría de los casos se buscó realizar la inseminación de dos yeguas por colecta. Cuando sólo se tuvo que servir a una yegua, se tomaba la decisión de usar diluyente o no, dependiendo de los resultados de la evaluación del semen pos-colecta. 
A partir del servicio de IA, las yeguas fueron controladas ecográficamente cada 24 $\mathrm{h}$ hasta la detección de un cuerpo hemorrágico $(\mathrm{CH})$, indicador de una ovulación reciente. Se realizó un segundo o tercer servicio por IA en las yeguas que no habían ovulado en el momento de la evaluación ecográfica. Para el grupo EX se calculó el tiempo en horas (h) desde la fecha y hora de aplicación de la hormona hasta la observación de un $\mathrm{CH}$ mediante el examen ultrasonográfico. Para el grupo CO se tomó como partida la fecha y hora del hallazgo de un FD de 38 a $40 \mathrm{~mm}$ para realizar el cálculo del tiempo de ovulación.

El diagnóstico de preñez se realizó entre los 13 y 15 días pos-ovulación mediante ultrasonido. El porcentaje de preñez se calculó con base al total de animales evaluados. Para el análisis del tiempo de ovulación (en horas) y del porcentaje de preñez se hizo uso del programa estadístico InfoSTAT, obteniéndose la estadística descriptiva; asimismo, se aplicó la prueba de «t» para muestras apareadas con un intervalo de confianza de $95 \%$.

Cuadro 1. Tiempo de ovulación en yeguas Caballo Peruano de Paso con o sin aplicación de acetato de deslorelina (ocho yeguas por grupo)

\begin{tabular}{|c|c|c|}
\hline $\begin{array}{l}\text { Tiempo de } \\
\text { ovulación } \\
\text { (horas) }^{1}\end{array}$ & $\begin{array}{c}\text { Control } \\
(\%)\end{array}$ & $\begin{array}{c}\text { Experimental } \\
(\%)\end{array}$ \\
\hline$\leq 48$ & 37.5 & 87.5 \\
\hline$>48-\leq 72$ & 50 & 12.5 \\
\hline$>72-\leq 96$ & 0 & 0 \\
\hline$>96-\leq 120$ & 12.5 & 0 \\
\hline Total & 100 & 100 \\
\hline
\end{tabular}

\section{Resultados y Discusión}

Solo tres $(37.5 \%)$ de las ocho yeguas evaluadas del grupo CO ovularon dentro de las $48 \mathrm{~h}$ a partir de la detección de un folículo dominante, en tanto que cinco ovularon entre 72 y 120 h después. En contraste, el 87.5\% (7/8) de las yeguas del grupo EX ovularon entre las 32 y $48 \mathrm{~h}$ pos-tratamiento (Cuadro 1). El $87.5 \%$ de eficacia de la deslorelina para inducir ovulación dentro de las $48 \mathrm{~h}$ de su administración es similar al trabajo de Farquhar et al. (2001), quienes obtuvieron 84 y $93 \%$ de yeguas evaluadas ovuladas dentro de los 2 o 3 días de tratamiento con Ovuplant (implante subcutáneo de Deslorelina), respectivamente. Por otro lado, en el estudio de BIOTEQ (2013) se reporta 99\% de eficacia para la producción de ovulación dentro de las 48 h. Asimismo, Finan et al. (2016) encontraron que el $93.75 \%$ de las yeguas ovularon dentro de las $48 \mathrm{~h}$ después de la aplicación de Bio-release Deslorelin (vial para administración intramuscular). Las diferencias observadas entre los estudios podrían deberse a un factor poblacional y/o ambiental (Boeta et al., 2006; Ramírez, 2006), nutricional o de raza.

Se calculó una media de $69.00 \pm 8.41 \mathrm{~h}$ (2.87 días) de intervalo a la ovulación para el grupo control y $43.13 \pm 4.48$ h (1.79 días) para el grupo experimental, habiendo diferencia estadística a favor del grupo experimental (Cuadro 2). Los resultados obtenidos demuestran que las yeguas tratadas con acetato de deslorelina evidencian una significativa reducción en el tiempo de ovulación desde la detección de un folículo dominante (38-40 $\mathrm{mm})$ Estos valores son similares a los reportados por BIOTEQ (2013), quienes obtuvieron un promedio de $86 \mathrm{~h}$ para su grupo control y de $42.5 \mathrm{~h}$ para el tratado con deslorelina, en tanto que Sauberli (2013) registró una media de 3.87 días para las que ovularon de manera espontánea y de 2.02 días para las yeguas que recibieron la hormona. 
Cuadro 2. Intervalo a la ovulación en yeguas Caballo Peruano de Paso con o sin aplicación de acetato de deslorelina (ocho yeguas por grupo)

\begin{tabular}{lccc}
\hline Grupo & Media & $\begin{array}{c}\text { Error } \\
\text { estándar }\end{array}$ & $\begin{array}{c}\text { Desv. } \\
\text { estándar }\end{array}$ \\
\hline Control & $69^{\mathrm{a}}$ & 8.4 & 23.8 \\
Experimental & $43^{\mathrm{b}}$ & 4.5 & 12.7 \\
\hline $\mathrm{a}, \mathrm{b}$ Diferentes letras dentro de columnas indica \\
\multicolumn{2}{l}{ diferencia significativa $(\mathrm{p}<0.05)$}
\end{tabular}

Cuadro 3. Número de yeguas Caballo Peruano de Paso con o sin aplicación de acetato de deslorelina (ocho yeguas por grupo), según el número de inseminaciones recibidas

\begin{tabular}{ccc}
\hline Servicios & $\begin{array}{c}\text { Control } \\
(\mathrm{n})\end{array}$ & $\begin{array}{c}\text { Experimental } \\
(\mathrm{n})\end{array}$ \\
\hline 1 & 4 & 6 \\
2 & 3 & 2 \\
3 & 1 & 0 \\
\hline Total & 8 & 100 \\
\hline
\end{tabular}

En las yeguas del grupo control fue necesario efectuar hasta 3 IA para aquellas que más tardaron en ovular (1/8), obteniendo un promedio de 1.75 IA por yegua. Por el contrario, el 75\% (6/8) de las yeguas del grupo experimental recibieron solo un servicio y a 2 de ellas se le realizaron 2 IA, obteniendo una media de 1.25 IA por animal (Cuadro 3), aunque sin diferencia estadística entre grupos. Estos resultados coinciden con Sauberli (2013), quien registró 1.21 IA por ciclo usando deslorelina en yeguas con folículos entre 35-39 mm en comparación con 1.39 IA en las yeguas no tratadas.
Pese a la ausencia de diferencias estadísticas entre grupos, tanto el presente estudio como los antecedentes citados comprueban que se disminuye el intervalo entre el folículo maduro y la ovulación con el uso del acetato de deslorelina, lo cual permite optimizar el uso de los padrillos y de las dosis de semen.

El 50\% (4/8) de las yeguas del grupo control y el $87 \%(7 / 8)$ de las tratadas con acetato de deslorelina quedaron preñadas, aunque no se observó diferencia estadística entre grupos por el bajo número de animales en estudio. Sin embargo, dichos resultados corroboran el reporte de Sauberli (2013), quién encontró que las yeguas tratadas estaban 2.1 veces más predispuestas a quedar preñadas si la ovulación era inducida. Por otro lado, dado que todas las yeguas estaban libres de problemas reproductivos y ambientales y, además, estaban en buena condición corporal, es muy probable que la diferencia en el porcentaje de preñez se deba al momento en que se efectuó la IA. Sieme (2003) sostiene que, para lograr tasas óptimas de preñez, es necesario que el semen sea depositado entre 12 horas antes o 6 horas después de la ovulación; en tanto que Newcombe y CuervoArango (2011) concuerdan que las tasas de preñez son mayores en yeguas inseminadas dentro de las $24 \mathrm{~h}$ previas a la ovulación o antes de las $16 \mathrm{~h}$ pos-ovulación. Es claro que dicha precisión es muy difícil de conseguir cuando la ovulación es espontánea, como se dio en el grupo control, salvo que se realicen múltiples montas o servicios de IA.

\section{Conclusión}

La administración de un tratamiento hormonal a base de acetato de deslorelina en el momento de hallazgo de un folículo dominante de 38-40 mm reduce considerablemente el tiempo de ovulación, permitiendo efectuar la inseminación artificial en el momento ade- 
cuado y, por lo tanto, reducir el número de servicios sin afectar la tasa de concepción.

\section{Literatura Citada}

1. Rodríguez I. 2010. Inseminación artificial o monta dirigida en la yegua. Argos Portal Veterinaria. [Internet]. Disponible en: http://argos.portalveterinaria.com/noticia/4662/articulos-archivo/inseminacion-artificial-o-monta-dirigida-en-la-yegua.html

2. Barrier-Battut I, Le Poutre N, Trocherie E, Hecht S, Grandchamp des Raux A, Nicaise JL, Vérin X, et al. 2001. Use of buserelin to induce ovulation in the cyclic mare. Theriogenology 55: 1679-1695. doi: 10.1016/S0093-691X(01)00512-X

3. Blanchar TL, Brinsko SP, Rigby SL. 2002. Effects of deslorelin or hCG administration on reproductive performance in first postpartum estrus mares. Theriogenology 58: 165-169. doi: 10.1016/S0093-691X(02)00912-3

4. Boeta M, Porras A, Zarco LA, AguirreHernández R. 2006. Ovarian activity of the mare during winter and spring at a latitude of $19^{\circ} 21^{\prime}$ north. J Equine Vet Sci 26: 55-58. doi: 10.1016/ j.jevs.2005.12.003

5. Camillo F, Pacini M, Panzani D, Vannozzi I, Rota A, Aria G. 2004. Clinical use of twice daily injections of buserelin acetate to induce ovulation in the mare. Vet Res Commun 28 (Suppl 1): $169-172$.

6. Davies MM. 2005. Fisiología de la reproducción de los équidos, cría y manejo de la yeguada. Zaragoza: Acribia. $426 \mathrm{p}$.

7. Farquhar VJ, McCue PM, Nett TM, Squires EL. 2001. Effect of deslorelin acetate on gonadotropin secretion and ovarian follicle development in cycling mares. J Am Vet Med Assoc 218: 749752. doi: 10.2460/javma.2001.218.749
8. Finan SA, Lamkin EL, McKinon AO. 2016. Comparative efficacy of BioRelease Deslorelin ${ }^{\circledR}$ injection for induction of ovulation in oestrus mares: a field study. Aus Vet J 94: 338-340. doi: 10.1111/avj. 12478

9. Fragoso CY. 2007. Inducción al estro en yeguas por medio de prostaglandinas. Tesis de Médico Veterinario Zootecnista. Michoacán: Univ. Michoacana de San Nicolás de Hidalgo. $61 \mathrm{p}$.

10. Galina C, Valencia J. 2008. Reproducción de los animales domésticos. $3^{\text {ra }}$ ed. México DF: Limusa. 582 p.

11. Henneke D, Potter GD, Kreider JL, Yeates DF. 1983. Relationship between condition score, physical measurements and body fat percentages in mares. Equine Vet J 15: 371-372. doi: 10.1111/ j.2042-3306.1983.tb01826.x

12. Maturana J, Droguett N, Ramirez H. s.f. Comparación entre gonadotrofina coriónica humana (hCG) y deslorelina en sus tiempos de acción, eficacia en inducir ovulación e incidencia de ovulaciones múltiples en yeguas Pura Raza Chilena. [Internet]. Disponible en: http:// slideplayer.es/slide/10738941/

13. McCue PM, Farquhar VJ, Carnevale EM, Squires EL. 2002. Removal of deslorelin (Ovuplant) implant $48 \mathrm{~h}$ after administration results in normal interovulatory intervals in mares. Theriogenology 58: 865-870. doi: 10.1016/S0093-691X(02)00923-8

14. McKinnon AO. 2009. Hormonal control of equine reproduction. In: $11^{\text {th }}$ Annual Resort Symposium of the American Association of Equine Practitioners. Australia: AAEP.

15. Melo CM, Papa FO, Puoli Filho JN, Araújo GH, Dell'Aqua Jr. JA, Alvarenga MA. 2012. Eficiência do acetato de deslorelina e do extrato de pituitária equina na indução da ovulação em éguas. Vet Zootec 19: 392-398.

16. Newcombe JR, Cuervo-Arango J. 2011. The effect of time of insemination with fresh cooled transported semen and 
natural mating relative to ovulation on pregnancy and embryo loss rates in the mare. Reprod Domest Anim 46: 678-681. doi: 10.1111/j.1439-0531.2010.01728.x

17. Ramírez J. 2006. Determinación del fotoperiodo sobre la actividad ovárica en yeguas durante el año, en diferentes haras, en los departamentos de Guatemala, Sacatepequez y Escuintla. Tesis de Médico Veterinario. Guatemala: Univ. de San Carlos de Guatemala. $97 \mathrm{p}$

18. Samper JC, Pycock JF. 2007. Section I: The normal female reproductive system. Chapter 5: The normal uterus in estrus. In: Samper JC, Pycock JF, McKinnon AO (eds). Current therapy in equine reproduction. USA: Elsevier.p32-35.
19. Sauberli D. 2013. The effectiveness and efficiency of ovulation induction agents in mares. MSc Thesis. Illinois: University of Illinois. $115 \mathrm{p}$.

20. Sieme H, Schäfer T, Stout TA, Klug E, Waberski D. 2003. The effects of different insemination regimes on fertility in mares. Theriogenology 60: 1153-1164. doi: 10.1016/S0093-691X(03)00113-4

21. Yoon M. 2012. The estrous cycle and induction of ovulation in mares. J Anim Sci Technol 54: 165-174.

22. Zamudio JV. 2005. Uso de prostaglandinas para sincronizar estro en yeguas criollas y sus efectos colaterales. Tesis de Médico Veterinario Zootecnista. Veracruz: Univ. Veracruzana. 49 p. 